\title{
Real-time remedial action against aperiodic small signal rotor angle instability
}

Weckesser, Johannes Tilman Gabriel; Jóhannsson, Hjörtur; Østergaard, Jacob

Published in:

IEEE Transactions on Power Systems

Link to article, DOI:

10.1109/TPWRS.2015.2404872

Publication date:

2016

Document Version

Peer reviewed version

Link back to DTU Orbit

Citation (APA):

Weckesser, J. T. G., Jóhannsson, H., \& Østergaard, J. (2016). Real-time remedial action against aperiodic small signal rotor angle instability. IEEE Transactions on Power Systems, 31(1), 387-396.

https://doi.org/10.1109/TPWRS.2015.2404872

\section{General rights}

Copyright and moral rights for the publications made accessible in the public portal are retained by the authors and/or other copyright owners and it is a condition of accessing publications that users recognise and abide by the legal requirements associated with these rights.

- Users may download and print one copy of any publication from the public portal for the purpose of private study or research.

- You may not further distribute the material or use it for any profit-making activity or commercial gain

- You may freely distribute the URL identifying the publication in the public portal

If you believe that this document breaches copyright please contact us providing details, and we will remove access to the work immediately and investigate your claim. 


\title{
Real-Time Remedial Action against Aperiodic Small Signal Rotor Angle Instability
}

\author{
Tilman Weckesser, Member, IEEE, Hjörtur Jóhannsson, Member, IEEE, \\ and Jacob Østergaard, Senior Member, IEEE
}

\begin{abstract}
This paper presents a method that in real-time determines remedial actions, which restore stable operation with respect to aperiodic small signal rotor angle stability (ASSRAS) when insecure or unstable operation has been detected. An ASSRAS assessment method is used to monitor the stability boundary for each generator in real-time. The ASSRAS boundary represents the condition when a generator reaches the maximum steady state active power injection. The proposed control method exploits analytically derived expressions for the ASSRAS boundary and other characteristic curves in the injection impedance plane to determine an active power redispatch among selected generators to restore stable and secure operation. Since the method is purely based on analytically derived expression, the computation of the remedial actions is fast and well suited for real-time operation. The method was tested on the IEEE 14-bus and the Nordic32 test systems where results show that the method can efficiently determine the required active power redispatch to avoid an imminent instability.
\end{abstract}

Index Terms-power system stability, power system control, power system generation redispatch, remedial action schemes

\section{INTRODUCTION}

$\mathbf{M}$ ODERN societies are highly dependent on a stable and secure operation of the power system. A continuously increasing share of power production based on renewable energy sources (RES) can be observed in numerous countries, where e.g. in Denmark the government's energy strategy states that $50 \%$ of the electricity consumption is to be supplied by wind power by 2020 [1]. The foreseen challenges associated with these goals are great as the future power system has to be securely operated and delivering energy at competitive prices.

The fluctuating nature of RES such as wind and solar radiation may cause rapid changes in future generation patterns, leading to rapid fluctuations of the power system's operating point. Existing offline and computationally demanding approaches for assessing stability and determination of remedial or preventive actions may become insufficient. Hence, a need for real-time approaches will arise for the future system [2].

Efforts have been made to meet the real-time requirements for the assessment of the future system. A method for online assessment of voltage stability is presented in [3], [4]. The method does not rely on a dynamic model to predict the system response, instead basic assumptions and simplifications are applied in the modeling process resulting in reduced computational burden. In [5] an element wise approach is

The authors are with the Center for Electric Power and Energy, Department of Electrical Engineering, Technical University of Denmark, 2800 Lyngby, Denmark. (email: jtgw@elektro.dtu.dk)

Research supported by the Danish Council for Strategic Research (DSF).

Manuscript received May 31, 2016. proposed for stability assessment where each individual assessment method analyzes a particular instability mechanism. [5] describes such a method to monitor the aperiodic small signal rotor angle stability (ASSRAS) of the individual generators and to determine their respective stability margin. This stability mechanism refers to the ability of each individual generator to produce sufficient steady state electromechanical torque. If this torque balance is upset, an aperiodic increase in rotor angle and a subsequent loss of synchronism can be observed.

The authors of [6] emphasize that efficient remedial action schemes (RAS) are an enabler for connecting more RES based generation. The real-world implementation of a Centralized Remedial Action Scheme (CRAS) system was described in [6]. The system executes corrective actions such as load or generation reduction to ensure reliable and safe system operation after fault occurrence. The automatic system-wide RAS arming system currently used in the power grid of British Columbia was described in [7]. The RAS arming patterns are determined periodically employing a transient stability analysis tool, which ensures security of the system. To achieve real-time performance, the system relies on a large case database build from extensive off-line planning studies.

A new methodology for determining the security region for operation of transmission systems is described in [8]. The calculation of the boundaries is done offline and can be used to identify efficient controls and remedial actions. The authors of [9] propose an adaptive damping control scheme, which uses online measurements to adapt the controller parameters to changing operating conditions. The initial parameters are determined offline using a set of nominal operating conditions. In [10] the authors compare two different approaches for damping inter-area oscillations and come to the conclusion that widearea control methods are more effective than local controls. The previous two paper determine controls for periodic small signal rotor angle stability, while the following method and the one proposed in this paper address ASSRAS.

In [11] the authors developed a method, which determines the necessary countermeasures to be applied to a number of loads to restore stability and security of the system with respect to the ASSRAS boundary. The method therefore alters the consumption pattern of load buses, which were identified to be the most effective locations for applying countermeasures.

This paper presents a new method to restore ASSRAS for which a patent has been submitted [12]. The investigated instability mechanism is a quasi steady state phenomena. Consequently, the assessment method as well as the developed remedial action method require that the system is in quasi 


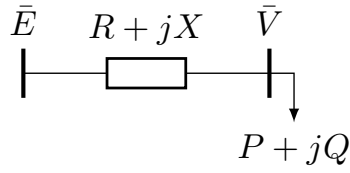

(a) Two bus system

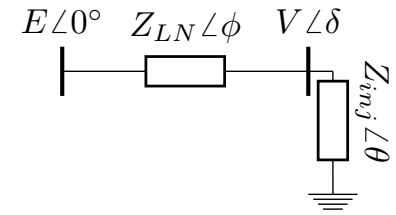

(b) Equivalent two bus system with injection impedance
Fig. 1. In [13] for this two bus system critical and characteristic curves were expressed in terms of the injection impedance

steady state. Most of the prior mentioned methods rely on extensive offline studies to determine the corrective control actions. In contrast to that, the proposed method is derived analytically and only requires online measurements of the current system condition. With these information, the method can fast and accurately determine the required control actions. The structure of the paper is as follows. In Section II, the ASSRAS assessment method is briefly described. The data provided by the assessment method are utilized in the developed remedial action method to compute corrective active power redispatch solutions, which is described in Sec. III. The method's capability to avoid an imminent collapse in voltage is presented in Sec. IV, where the results from two test systems are presented. Finally, in Sec. V the presented method and results are discussed.

\section{BACKGROUND}

\section{A. Algebraic Expressions for Critical Transmission Limits}

Reference [13] describes the mapping of some characteristic curves from a PQV-surface of a two bus system into the injection impedance plane (see Fig. 1). For such a system the relationship of the voltage magnitudes in both ends ( $E$ and $V)$ and the active $\&$ reactive power $(P$ and $Q)$ becomes:

$$
V^{4}+V^{2}\left(2(R P+X Q)-E^{2}\right)+\left(R^{2}+X^{2}\right)\left(P^{2}+Q^{2}\right)=0
$$

where $R$ and $X$ are the resistance and reactance of the line. In [13] it is shown, by manipulation of (1), that the condition for maximum deliverable power to the receiving end, under the assumption of constant $E$ and $V$, is represented by a circle in the injection impedance plane, which is in polar coordinates:

$$
Z_{i n j}=-Z_{L N} \sin (\theta) / \sin \phi
$$

Here, $Z_{i n j} \angle \theta$ represents the complex injection impedance and $Z_{L N} \angle \phi$ the impedance of the line where $Z_{L N}=\sqrt{R^{2}+X^{2}}$. Furthermore in [13], it was shown that lines of constant voltage magnitude $V$ and voltage angle $\delta$ (the angle between $E$ and $V$ ) map as circles in the injection impedance plane. For later use the equations describing these characteristic curves are stated at this point. The curves of constant voltage magnitude satisfy:

$$
Z_{i n j}=r_{0} \cdot \cos (\theta-\varphi) \pm \sqrt{r^{2}+r_{0}^{2} \cdot \sin ^{2}(\theta-\varphi)}
$$

where

$$
r=\frac{E V \sqrt{R^{2}+X^{2}}}{V^{2}-E^{2}} ; \quad r_{0}=\frac{V^{2} \sqrt{R^{2}+X^{2}}}{V^{2}-E^{2}} ; \quad \tan \varphi=\frac{-X}{-R}
$$

From [13], the curve for constant voltage angle $\delta$ is given by:

$$
Z_{i n j}=-\left(Z_{L N} / \sin \delta\right) \cdot \sin (\delta+\phi-\theta)
$$

\section{B. Method for Real-Time Assessment of ASSRAS}

In [5] a method is presented to quickly assess the ASSRAS of all system generators. In Fig. 2, a block diagram of the employed method is shown to visualize the different steps carried out to assess stability. The algebraically derived expression for maximum injection of active power in (2) is exploited to formulate an algebraic assessment criterion which enables stability assessment in linear time [14]. The ASSRAS boundary of a generator is given by:

$$
Z_{i n j, i}=-\left(Z_{t h, i} \sin (\theta)\right) /\left(\sin \phi_{t h, i}\right)
$$

where $Z_{i n j, i} \angle \theta=\bar{Z}_{i n j, i}$ is the complex injection impedance seen from the $i^{\text {th }}$ generator's node of constant steady state voltage magnitude and $Z_{t h, i} \angle \phi_{t h, i}$ is the complex Thévenin network impedance seen from the same node. In [15] it was shown that the Thévenin equivalent parameters seen from each generator can be directly computed from the network admittance matrix and a system snapshot. The authors of [14] presented a test case with 7917 buses and 1325 voltage control nodes and demonstrated that all the Thévenin equivalent parameters could be computed within $2.5 \mathrm{~ms}$.

Eq. (5) appears as a circle in the injection impedance plane and the generator's ASSRAS is determined from its value of $\bar{Z}_{i n j, i}$. A value of $\bar{Z}_{i n j, i}$ outside the circle represents stable operation while a value inside represents unstable operation. An unstable operation is characterized by the condition when a small increase of the steady state rotor angle $\delta$ of a given generator reduces its active power output. While during stable operation, an increase in $\delta$ increases the generator's active power output. The assessment method determines whether a steady state equilibrium point exists between the mechanical power applied to a given generator and its active power output. If a disturbance causes a loss of the generator's steady state equilibrium point, the generator will begin to lose synchronism in a process that may take 10's of seconds and up to a few minutes to evolve [5] [15]. In order to track the stability condition of a generator in real-time, system snapshots need to be available at a sampling rate equivalent to the repetition rate of measurements provided by PMUs or from a fast state estimator. The sampling rate of the snapshots should be sufficient to trace the movement of the operating points and a higher rate allows to choose lower security limits.

As the cause of aperiodic small signal rotor angle instability is the lack of existence of an equilibrium point between the mechanical and electrical power of a given generator, an intuitive choice of a counter action is to lower mechanical power applied to the critical machine to restore an equilibrium.

Stability Margin In [5] the authors showed that the distance of the operating point (OP) of a generator to the stability boundary is a measure of the distance to instability and that it can be expressed in terms of various variables. When representing a generator by a voltage source $V \angle \delta$ and the remaining grid by a Thevenin equivalent corresponding to a voltage source with magnitude $E_{t h}$ and an impedance $Z_{t h} \angle \phi_{t h}$, then its power injection $P_{i n j}$ is:

$$
P_{i n j}=\left(E_{t h} V / Z_{t h}\right) \cos \left(\delta+\phi_{t h}\right)-\left(V^{2} / Z_{t h}\right) \cos \left(\phi_{t h}\right)
$$




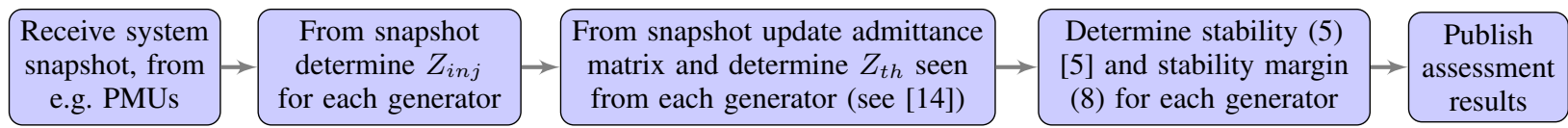

Fig. 2. Function blocks of the ASSRAS assessment method [5]

\begin{tabular}{|c|c|c|c|c|}
\hline $\begin{array}{l}\text { Imminent instability } \\
\text { detected with } \\
\text { ASSRAS method } \\
\text { (see II-B \& III-A) }\end{array}$ & $\begin{array}{l}\text { Check if system } \\
\text { in quasi steady } \\
\text { state (see III-B) }\end{array}$ & $\begin{array}{l}\text { Determine new } \\
\text { stable operating } \\
\text { point (see III-E) }\end{array}$ & $\begin{array}{c}\text { Determine available } \\
\text { power reserves } \\
\text { (see III-F) }\end{array}$ & $\begin{array}{c}\text { Generate redispatch } \\
\text { solutions and } \\
\text { identify preferable } \\
\text { solution (see III-G) }\end{array}$ \\
\hline
\end{tabular}

Fig. 3. Function blocks of the proposed remedial action method

Under the assumption of constant voltage magnitudes at nodes of power injection and freezing of all other phase angles, the power injection of a particular generator becomes a function of solely the phase angle $\delta$ and is maximal at the angle $\delta=180^{\circ}-\phi_{t h}$, which represents the ASSRAS boundary. Consequently, the maximum power injection $\hat{P}_{i n j}$ can be expressed as:

$$
\hat{P}_{i n j}=-E_{t h} V / Z_{t h}-\left(V^{2} / Z_{t h}\right) \cos \left(\phi_{t h}\right)
$$

This allows to define a stability margin in percentage of the maximum power injection $\% \Delta P_{i n j}$.

$$
\% \Delta P_{i n j}=\frac{\cos \left(\delta+\phi_{t h}\right)+1}{1+\frac{V}{E_{t h}} \cos \phi_{t h}} \cdot 100 \%
$$

\section{Relative electrical distance}

In [16], the authors describe an approach to determine the relative electrical distance between load buses and generator buses. For that purpose the authors utilize the network admittance and the linear algebraic network equations.

$$
\left[\begin{array}{c}
\mathbf{I}_{G} \\
\mathbf{I}_{L}
\end{array}\right]=\left[\begin{array}{ll}
\mathbf{Y}_{G G} & \mathbf{Y}_{G L} \\
\mathbf{Y}_{L G} & \mathbf{Y}_{L L}
\end{array}\right]\left[\begin{array}{l}
\mathbf{V}_{G} \\
\mathbf{V}_{L}
\end{array}\right]
$$

where the complex current and voltage vectors at the generator and load buses are represented by $\mathbf{I}_{G}, \mathbf{I}_{L}, \mathbf{V}_{G}$ and $\mathbf{V}_{L}$. The sub-matrices $\mathbf{Y}_{G G}, \mathbf{Y}_{G L}, \mathbf{Y}_{L G}$ and $\mathbf{Y}_{L L}$ are the corresponding parts of the network admittance matrix.

The authors showed that the sub-matrices can be used to determine the relative electrical distance of the load buses to the generator buses in the system, which can be computed as follows.

$$
\left[R_{L G}\right]=1-a b s\left[F_{L G}\right]=1-a b s\left(\left[\mathbf{Y}_{L L}\right]^{-1}\left[\mathbf{Y}_{L G}\right]\right)
$$

The values in each column of the resulting matrix contain a measure of the relative electrical distance between a load bus and the respective generator.

\section{METHOD FOR REAL-TIME DETERMINATION OF REMEDIAL ACTIONS}

This section describes a method capable of determining a generator redispatch solution to circumvent ASSRA instability in real-time. Figure 3 shows the function blocks of the proposed method.

\section{A. Trigger and security margins}

It is preferable that the remedial actions are executed before the system becomes unstable. Hence, a trigger margin is introduced that corresponds to a percentage of the maximum power injection and represents the stability margin threshold below which the remedial action method is executed. To find a new set point and to limit how much the remaining generators can contribute to the remedial action, a second threshold is introduced, called security margin $m_{s e c}$, which defines the security boundary. In this approach, it was assumed that the voltage at the node of power injection remains constant during the remedial action. This assumption is valid, because the node of power injection is chosen to be at the terminal of the generator or behind the synchronous reactance depending on the respective excitation system of the generator. Due to the constant voltage magnitude the power injection of the generator is solely determined by its voltage angle, see (6). Consequently, the curve corresponding to the trigger and the security margin are represented by a curve of constant voltage angle in the injection impedance plane (see (4) and Fig. 4).

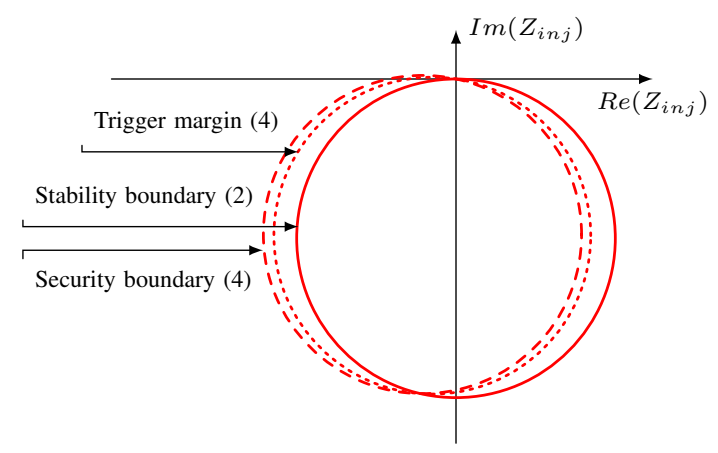

Fig. 4. Injection impedance plane displaying the aperiodic small signal rotor angle stability boundary (solid circle), the security boundary (dashed circle) and the circle corresponding to the trigger margin (dotted circle)

The selection of appropriate margin thresholds is a tradeoff between level of system security and additional constraints on the generator's capacity. Since the requirement of a greater margin directly affects the maximum power that a generator can provide to the system and, hence, may lead to an economical loss. Therefore, a balance between required margins for secure system operation and economically efficient operation of the generators has to be found. The margins may be different in each individual power system and it is suggested that the system operators choose them based on their experience and from offline studies of a selection of aperiodic small signal rotor angle stable and unstable contingencies. 
The assessment method analyses the ASSRAS of the system during quasi steady state conditions. However, a crossing of the stability boundary is acceptable in dynamic transition periods from one to another steady state equilibrium point. Consequently, the remedial action method should only be executed, when the system is in quasi steady state and the generators stability margin has fallen below the trigger margin.

\section{B. Quasi steady state and error estimation}

Since the method is based on the assumption of constant voltage magnitude at the nodes of power injection, the voltage magnitudes at these nodes can be used to determine the steadiness of the system. It is assumed that the system is in quasi steady state, when the maximum power injection at a node of constant voltage magnitude can be determined with satisfactory accuracy. Therefore, at a node with assumed constant voltage magnitude the measured voltage magnitudes provided by a PMU are stored for a certain period. Under the assumption that the Thevenin voltage has constant voltage magnitude, the maximum power injection at the node can be computed as a function of the measured voltage magnitudes $V\left(t_{n}\right)$ using (7). When extracting the maximum $V_{\max }$ and minimum $V_{\min }$ of the voltage magnitude in the period of concern, the corresponding maximum error of the computed maximum power injection $\Delta \% P_{i n j, e r r}$ can be determined.

$$
\Delta \% P_{i n j, e r r}=\frac{\hat{P}_{i n j}\left(V_{\max }\right)-\hat{P}_{i n j}\left(V_{\min }\right)}{\hat{P}_{i n j}\left(V\left(t_{n}\right)\right)} 100 \%
$$

In this paper the acceptable error was chosen to be $0.5 \%$.

\section{N-1 operating point}

The discussed assessment method assumes either the voltage magnitude at the terminal of the machine to be constant or at the internal node behind the synchronous reactance $X_{s}$. If saliency is neglected, $X_{s}$ is equal to the reactance in the $d$ axis $X_{d}$ and $q$-axis $X_{q}$. Hence, under the assumption that the complex voltage $\bar{E}_{t}$ and the complex current $\bar{I}_{t}$ at the terminal of the machine are monitored, then the internal voltage $\bar{E}_{q}$ can be computed as follows.

$$
\bar{E}_{q}=\bar{E}_{t}+j X_{d} \bar{I}_{t}
$$

For a salient machine the assumption of constant voltage behind $X_{d}$ introduces an error, which was investigated in [15]. The results showed that the assumption leads to very small deviations and slightly more conservative assessment results. A machine equipped with an automatic voltage regulator (AVR) will keep the voltage magnitude at the terminal constant, unless the excitation exceeds its limit and the over excitation limiter (OEL) is activated. The activation will cause that the field current is kept constant at a limit value and, hence, the voltage at the terminal can no longer be assumed constant. However, under such conditions the voltage magnitude at the internal node behind $X_{d}$ can be assumed constant. During AVR operation with constant voltage magnitude at the terminal, the stability of the $N-1 \mathrm{OP}$, which assumes constant voltage magnitude at the internal node, can be monitored simultaneously with the actual OP. This allows to provide information on the stability condition of the system in case of activation of an OEL of a machine. Furthermore, it allows to determine control actions guaranteeing secure operation in case of activation of an OEL. If remedial actions are considered for $N-1$ OPs, the trigger and security margins may be chosen lower than for actual OPs, since a stability boundary crossing of a $N-1$ OP only corresponds to an insecure, but not unstable situation.

\section{Assumption of constant voltage magnitude}

In this section it is described at which node the voltage magnitude is assumed to be constant during the remedial action. Table I shows where the voltage magnitude is assumed to be constant depending on the generator's excitation system, the state of the OEL and the type of the OP, whose stability margin fell below the trigger margin or rather is imminent to experiencing instability.

TABLE I

LOCATION OF NODE OF CONSTANT VOLTAGE MAGNITUDE DURING REMEDIAL ACTION IN DIFFERENT GENERATOR CONFIGURATION AND OPERATION CONDITIONS

\begin{tabular}{|c|c|c|c|}
\hline Excitation system & OEL & Actual OP & $N-1$ OP \\
\hline Manually & - & behind $X_{d}$ & - \\
\hline \multirow{2}{*}{ AVR with OEL } & inactive & at terminal & at terminal \\
\cline { 2 - 4 } & active & behind $X_{d}$ & - \\
\hline
\end{tabular}

In the simple case that the machine is manually excited, the voltage magnitude is assumed to be constant behind $X_{d}$ and may be computed with (12). In the more complex case, where the machine is equipped with an AVR the location of the node of constant voltage during the remedial action is dependent on the state of the OEL. If the OEL is inactive and, hence, the AVR keeps the voltage magnitude at the machine terminal constant, then the voltage magnitude during the remedial action is assumed to be constant at the terminal independent on the type of OP that is imminent unstable/insecure. If the OEL is activated, the node of constant voltage magnitude moves behind $X_{d}$ and, consequently, this is also the node, where the voltage magnitude is assumed to be constant throughout the control action.

\section{E. Computation of new stable and secure operating point}

1) Imminent unstable node is actual operating point: In the case that the margin of the actual OP of a generator fell below the trigger margin, the power output of the generator has to be reduced to move the OP back into the region of secure operation. Since the desired security margin $m_{s e c}$ is known as a percentage of the maximum power injection and under the assumption that the voltage magnitude ratio $V / E_{t h}$ remains constant throughout the remedial action, (8) can be used to compute the voltage angle at the new secure OP.

$$
\delta^{*}=\arccos \left[\frac{m_{s e c}}{100 \%}\left(1+\frac{V}{E_{t h}} \cos \left(\phi_{t h}\right)\right)-1\right]-\phi_{t h}
$$

Subsequently, with the new voltage angle $\delta^{*}$, the new active power injection at the secure OP can be determined utilizing (6). Then the necessary power reduction can be determined as the difference between power injection at the current voltage angle $\delta$ and the new secure voltage angle $\delta^{*}$. Figure 5 depicts the determination of the new secure OP in the injection impedance plane. 


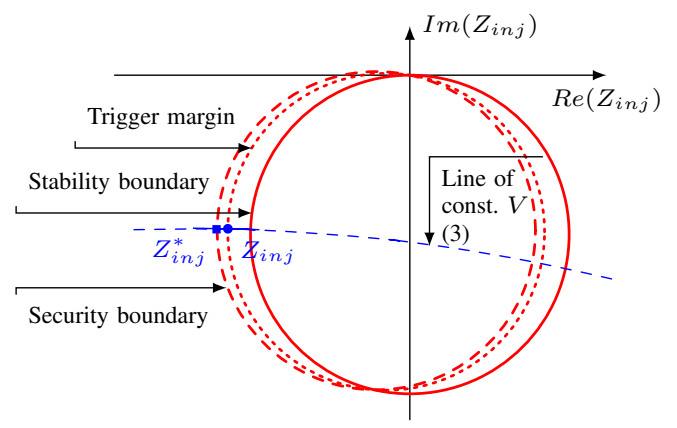

Fig. 5. Graphical representation of the computation of a new secure operating point for the case of imminent instability due to an actual operating point

The injection impedance $Z_{i n j}$ (blue dot) of the respective generator crossed the trigger boundary, which executed the method. Following, the new angle $\delta^{*}$ was computed using (13). The new injection impedance $Z_{i n j}^{*}$ of the generator is then found by determining the intersection of the curve of constant voltage magnitude and the line of constant voltage angle corresponding to $\delta^{*}$.

2) Imminent unstable node is $N$ - 1 operating point: In order to operate the system in a secure manner, it is desirable that the $N-1$ OP of a machine is also in the stable and secure operating region. The computation of the needed power reduction differs from the calculations in Sec. III-E1, since the voltage magnitude at the internal node, which is used to compute the $N-1 \mathrm{OP}$, cannot be assumed to be constant throughout the process. It is assumed that also in this case the AVR of the generator keeps the voltage magnitude at the terminal constant and, hence, the corresponding characteristic curve of constant voltage magnitude can be utilized.

In the injection impedance plane, the distance between the injection impedance of the $N-1$ OP and the injection impedance of the actual OP is purely imaginary and equal to $X_{d}$. This allows to map characteristic curves of the actual to the $N-1$ OP (see Fig. 6).

The new secure OP of the $N-1 \mathrm{OP}$ can be found as the intersection of the circle representing the security boundary of the $N-1$ OP with the circle corresponding to the curve of constant voltage magnitude of the actual operating point mapped to the $N-1$ OP (see Fig. 6).

The equation for curves of constant voltage magnitude as described in (3) can be rewritten as:

$$
\bar{Z}_{\text {inj,const. }|V|}=r_{0} \cdot e^{j \phi_{t h}}+r \cdot e^{j \theta}
$$

where $r$ is the radius of the circle, the distance from the origin to the centre of the circle is $r_{0}$ and the angle between real axis and a line through the origin and centre of the circle is $\phi_{t h}$.

This circle of constant voltage magnitude can be mapped to the $N-1$ OP by subtracting $j X_{d}$.

$$
\bar{Z}_{i n j, \text { const. }|V|, T}=r_{0} \cdot e^{j \phi_{t h}}+r \cdot e^{j \theta}-j X_{d}
$$

Curves of constant voltage angle in the injection impedance are described by (4) and this shows that all the circles of constant voltage angle intercept the origin. Consequently, the term in front of the sine corresponds to the diameter of the circle. This allows to determine the radius of the circle as well as the angle between real axis and the line through origin and centre of the circle. The equation can be rewritten and expressed in terms of the Thevenin parameters as follows:

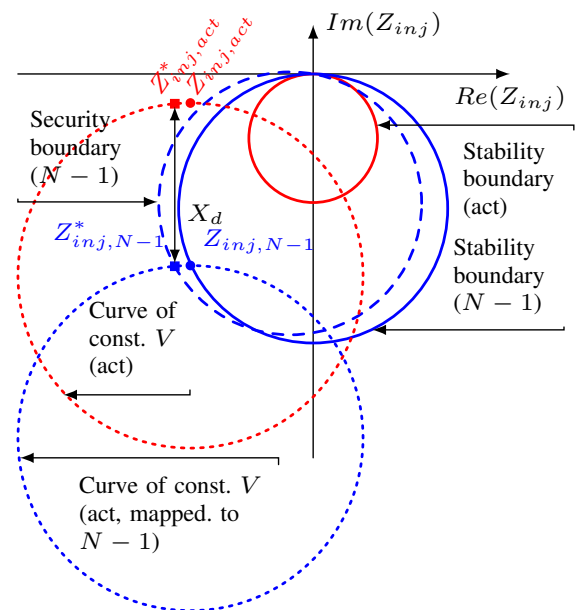

Fig. 6. Graphical representation of the computation of a new secure operating point for the case of imminent instability due to an $N-1$ operating point

$$
\bar{Z}_{i n j, c o n s t . \delta}=\frac{Z_{t h}}{2 \sin \delta}\left(e^{j\left(\delta+\phi_{t h} \pm \pi / 2\right)}+e^{j \theta}\right)
$$

Knowing the desired stability margin $m_{\text {sec }}$ and after computation of the current voltage magnitude at the internal node of the machine, the new secure voltage angle of the $N-1$ OP can be determined utilizing (13). Subsequently, the circle corresponding to the curve of constant voltage angle can be computed using (16). The intersection of the circle of constant voltage angle described by (16) and the mapped circle of constant voltage magnitude of the actual OP described by (15) gives the new secure $N-1$ OP in terms of the injection impedance $Z_{i n j, N-1}^{*}$. The corresponding new actual OP can be computed from the new actual injection impedance $Z_{i n j, a c t}^{*}$, which can be computed by adding the synchronous reactance to $Z_{i n j, N-1}^{*}$ (see Fig. 6). Since the voltage magnitude in the actual OP was assumed to be constant, the active power injection in the new and secure OP can be computed and the necessary active power reduction can be determined.

\section{F. Computation of available power reserves}

After the required power reduction was calculated the available power resources of the remaining generators have to be determined to eventually propose a power redispatch solution. The available resources are calculated, while respecting the following constraints. The "new" power injection should not

- exceed the $\hat{P}_{i n j}$ plus the respective security margin

- exceed nominal power of the generator plus a sec. margin

- move the corresponding $N-1$ OP into an insecure or unstable position. Hence, also for the $N-1$ OP a security margin should be maintained.

The power reserve with respect to the nominal power of the generator can directly be computed knowing the security margin and the machine parameter.

In the following, the power reserve computation for an actual OP and for an actual OP with respect to its $N-1$ OP are presented.

1) Power reserve of an actual operating point: The available power reserve $\Delta P_{i n j, r e s}$ of a generator with respect to its maximum power injection can simply be computed from the difference between the current stability margin $\% \Delta P_{i n j}$ 
(8) and the pre-determined security margin $m_{s e c}$, since both are expressed as percentages of $\hat{P}_{i n j}(7)$.

$$
\Delta P_{\text {inj,res }}=\left(\% \Delta P_{\text {inj }}-m_{\text {sec }}\right) / 100 \% \cdot P_{\text {inj,max }}
$$

2) Power reserve of an actual operating point with respect to corresponding $N-1$ operating point: In order to compute the power reserve an approach similar to the one in section III-E2 was taken. For that purpose the characteristic curves derived in [13] were exploited (see Fig. 7).

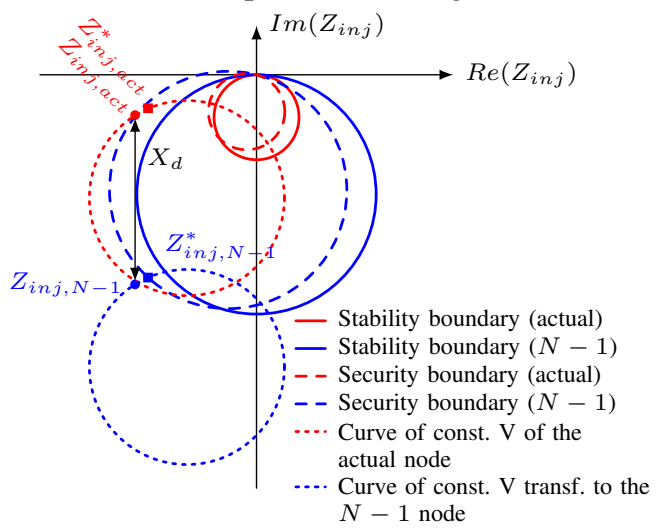

Fig. 7. Determining the power reserves of the actual operating point with respect to the maximum power injection of the corresponding $N-1 \mathrm{OP}$

The first step in determining the available power reserve is to calculate the phase angle corresponding to the security margin of the $N-1$ OP utilizing (13). Then the respective curve of constant phase angle (blue dashed curve in Fig. 7) can be computed with (16).

Because of the preventive action the voltage magnitude of the $N-1$ OP may change, but the voltage magnitude at the actual OP is assumed to remain constant. Consequently, the curve of constant voltage magnitude can be used to determine the available power reserve. The curve of constant voltage magnitude at the actual OP (red dotted curve in Fig. 7) is computed employing (15). Then this curve is transferred to the $N-1$ OP by adding $j X_{d}$ (blue dotted curve). The injection impedance that respects the security margin of the $N-1 \mathrm{OP}$ can be found as the intersection of the blue dotted and blue dashed circle. This impedance is transferred back to the actual $\mathrm{OP}$ and the available power reserve can be computed.

\section{G. Determination of redispatch solutions}

The preventive action comprises the power reduction at a particular generator to restore its stability (see Sec. III-E) and the increase of power generation of one generator or a group to counterbalance the power reduction. Depending on the determined power reserves of the remaining generators (see Sec. III-F), a variety of redispatch solutions can be identified employing different criteria for generator selection and for sharing of the required power increase between several generators. Possible generator selection criteria can be size of the individual power reserve, power margin, electrical distance to the generator in distress or consensus in served loads. Criteria for computing a share size for a particular supporting generator may be determined e.g. by the size of the generator or its available power reserve.

In the implementation presented in this paper, the generator or the group of generators to counter balance the power reduction are chosen corresponding to there relative electrical distance to the generator in distress. The idea is to redispatch the reduced power to the electrically close generators. For that purpose indices representing the relative electrical distance are computed with an approach based on the one described in Sec. II-C and [16]. The approach allows to determine the relative electrical distance of an internal machine node behind the synchronous reactance to an terminal of another machine. In order to utilize the method, the extended and augmented grid admittance matrix was assembled as follows.

$$
\mathbf{Y}=\left(\begin{array}{c|c}
N \times N & N \times M \\
\hline M \times N & M \times M
\end{array}\right)=\left[\begin{array}{ll}
\mathbf{Y}_{n n} & \mathbf{Y}_{n g} \\
\mathbf{Y}_{n g}^{T} & \mathbf{Y}_{g g}
\end{array}\right]
$$

where $N$ is the number of buses and $M$ the number of generators in the system. The matrix is augmented by the load admittances and the synchronous reactances of the generator. Furthermore, it is extended to the internal nodes of the generators. The $N \times N$ sub-matrix $\mathbf{Y}_{n n}$ is the regular grid admittance of the power system augmented by the load admittance and the synchronous reactances of the generators. The $M \times M$ matrix $\mathbf{Y}_{g g}$ is the sub-matrix containing all the entries due to the additional internal nodes, which were included due to the chosen representation of synchronous machines. The $N \times M$ matrix $\mathbf{Y}_{n g}$ is a sub-matrix, which is linking the internal nodes of the generators to the system buses corresponding to the generator terminals, and $\mathbf{Y}_{n g}^{T}$ is its transpose.

After dividing the grid matrix into the sub-matrices according to [16], the relative electrical distance of the internal nodes to the remaining nodes in the system can be calculated as follows.

$$
\left[R_{n g}\right]=1-a b s\left[F_{n g}\right]=1-a b s\left(\left[\mathbf{Y}_{n n}\right]^{-1}\left[\mathbf{Y}_{n g}^{T}\right]\right)
$$

The values in each column of the resulting matrix now contain a measure of the relative electrical distance between an internal node of the respective generator to its own terminal, to the terminals of the other generators and the remaining system nodes. Each entry has an offset due to the electrical distance between internal node and terminal, which can be corrected by subtracting the respective relative electrical distance between internal node and generator terminal from all the remaining entries in the particular column.

In the case that the total available power reserves are not sufficient to perform the required redispatch, an emergency solution is proposed, which aims at moving the OP of the critical generator as far as possible away from the stability boundary and towards the security boundary without jeopardizing stability and security of the remaining generators. For that purpose, the power reserves of the non-critical generators are computed as described in Sec. III-F and the total reserve $\Delta P_{\text {res,total }}$ as the sum of all reserves is computed, where $N C$ is the set of non-critical generators.

$$
\Delta P_{\text {res,total }}=\sum_{N C} \Delta P_{i n j, r e s}
$$

As stated before, in this case the total reserves are less than the needed power reduction computed in Sec. III-E. However, in order to stabilize the critical generator, its power injection is reduced by $\Delta P_{\text {res,total }}$, which can be counterbalanced by the remaining generators without putting them at risk. 


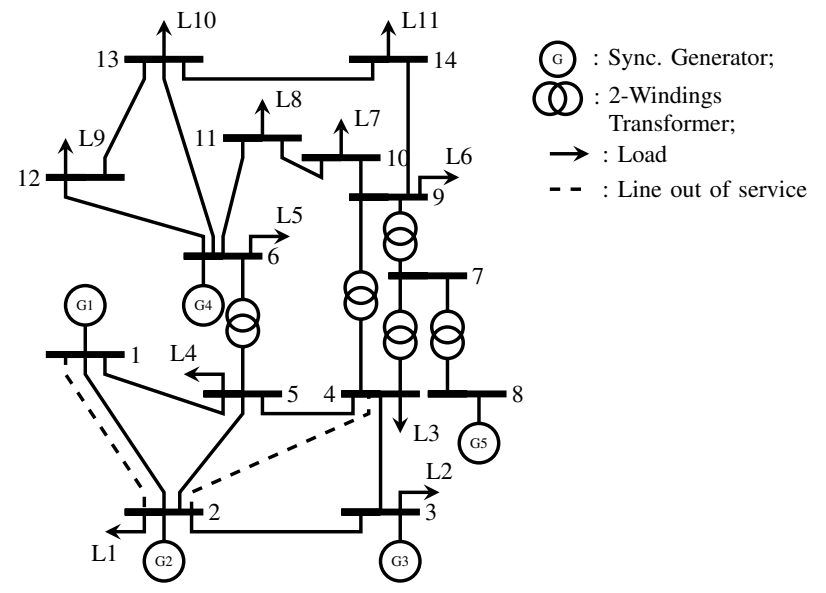

Fig. 8. One-line diagram of the modified IEEE 14-bus test system.

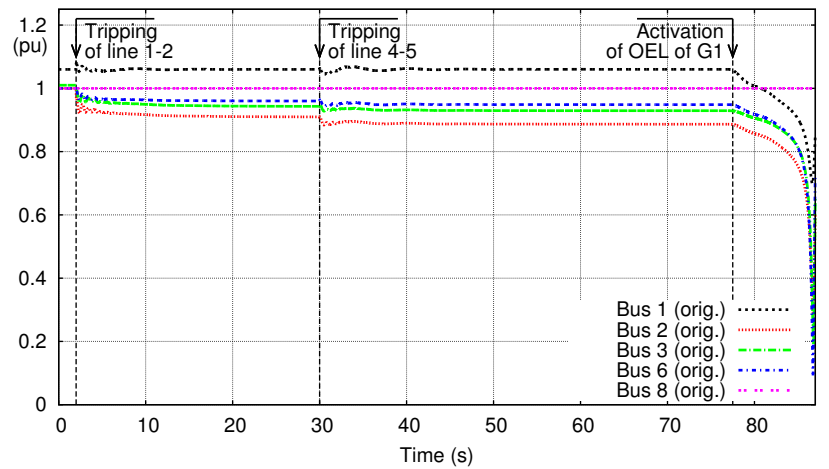

Fig. 9. IEEE 14-bus: Instability scenario: Generator bus voltages

\section{RESUlts}

\section{A. Scenario 1: N-1 OP crosses stability boundary}

1) Test System: A modified version of the IEEE 14-bus test system [17] was used. Modifications of the original system were amongst others the following. The three condensers in the system were replaced by generators to allow an active power redispatch. Bus 8 was considered to be an infinite bus and the generator connected to it represents a strong external grid. The synchronous machines G2-G4 are manually excited. The generators G1 and G5 are equipped with automatic voltage regulator (AVR), over excitation limiter (OEL) and power system stabilizer (PSS). A one-line diagram of the test system can be found in Fig. 8 .

2) Unstable scenario: In this scenario the system was highly loaded and in stressed conditions. To provoke instability, a sequence of contingencies were applied (see Fig. 9). The results of the time domain simulation were used to generate synthetic PMU measurements, which were fed to the assessment method. After the first and second disturbance, each corresponding to a loss of a transmission line, the system seems to reach a new quasi steady state (see Fig. 9). However, at the new OP the excitation voltage of G1 is too high and the OEL is activated after a pre-set delay. This last disturbance led to a loss of synchronism of the respective generator and, furthermore, to a collapse in voltage in the system.

The stability assessment results, when applying the ASSRAS method [5], are shown in Fig. 10. The figure shows a

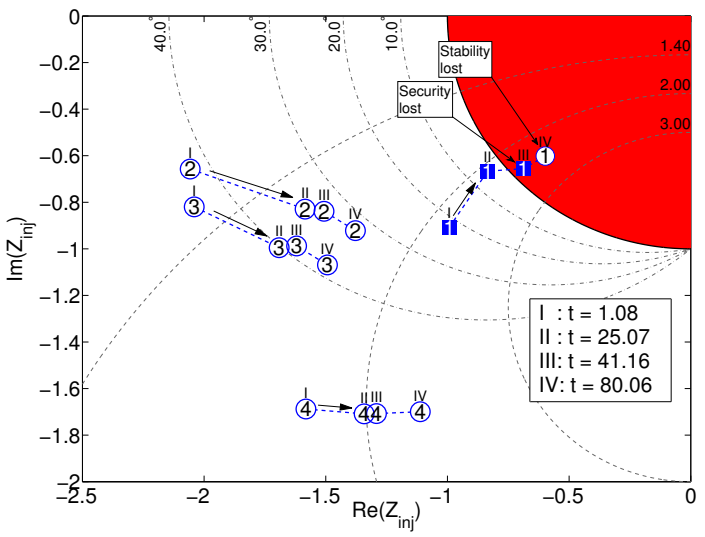

Fig. 10. IEEE 14-bus: Stability assessment results: Pre-fault (1.08 s), after first disturbance loss of line 1-2 (25.07 s), after second disturbance loss of line 4-5 (41.16 s), after third disturbance activation of OXL of G1 (80.06 s). Arabic numbers refer to the number of the generator, e.g. "1" refers to G1.

magnified detail of the normalized injection impedance plane. Here, the actual OP of a generator is depicted by a blue circle and the $N-1 \mathrm{OP}$ is indicated by a blue filled square. The colour of the large circle, which represents the stability boundary, indicates the stability state of the system, where green corresponds to a stable state and red to an unstable state. The state is assessed to be insecure if an OP has crossed the security margin or a $N-1$ OP has crossed the stability boundary, but the OEL is not yet activated.

Figure 10 shows the stability assessment results at four time instances labelled with the Roman numerals I-IV. In the prefault condition (see Fig. $10 \mathrm{I}$ ) the system is in steady-state. The first disturbance leads to a considerable reduction of the stability margin of the actual OPs of G2 and G3 and the $N-1$ OP of G1, but the system remains stable and secure (see Fig. 10 II). After the second disturbance and when a new quasi steady state is reached, the method identifies that the $N-1$ OP of G1 crossed the stability boundary (see Fig. 10 III). However, as long as the OEL of the generator is not activated the system remains stable, but is no longer considered secure. Consequently, the third disturbance, which corresponds to the activation of the OEL of G1, leads to an unstable state and, eventually, to a collapse in voltage (see Fig. 10 IV and Fig. 9).

The results show that the assessment method could detect an insecure operation of the system approximately $40 \mathrm{~s}$ before the actual collapse in voltage occurred.

3) Scenario with remedial action: In the following the same instability scenario is discussed, but this time, when insecure operation is detected, then the remedial action method described in Sec. III is triggered and a power redispatch is determined and executed to bring the system back into a secure state. Offline simulation of the test system showed, that for an $N-1 \mathrm{OP}$, the security and trigger margin can be chosen low with $0.5 \%$ and $0.1 \%$ of $\hat{P}_{i n j}$.

The stability margin of the $N-1 \mathrm{OP}$ of G1 had fallen below the trigger margin and the system reached a quasi steady state (see Sec. III-B) at the time instance shown in Fig. 10 III. Consequently, the remedial action method was executed and a redispatch as shown in Table II was determined. It should be noted, that generator G5 was not considered for the redispatch, 
TABLE II

ACTIVE POWER RESERVES AND RE-DISPATCH SOLUTION

\begin{tabular}{|l|c|c|c|c|}
\hline Generator: & 1 & 2 & 3 & 4 \\
\hline$\Delta P_{\text {reserve }}[M W]:$ & - & 9.7 & 9.7 & 4.9 \\
\hline$P_{\text {before }}[M W]:$ & 179.8 & 64.9 & 64.9 & 20.0 \\
\hline Size $[M V A]:$ & 200.0 & 75.0 & 75.0 & 25.0 \\
\hline$\hat{P}_{\text {inj }}$ in $[M W]:$ & 179.5 & 76.3 & 80.9 & 33.3 \\
\hline$\Delta P_{R E M}[M W]:$ & -22.4 & 9.0 & 9.0 & 4.4 \\
\hline$P_{\text {after }}[M W]:$ & 157.4 & 73.9 & 73.9 & 24.4 \\
\hline
\end{tabular}

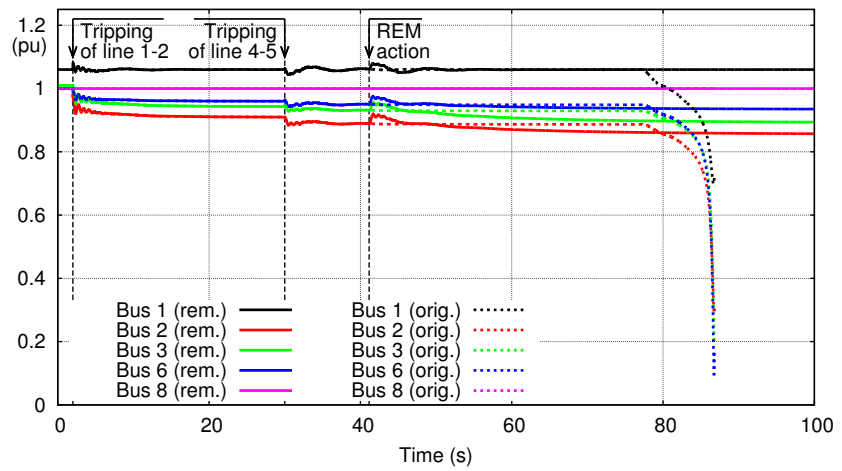

(a) Stabilized scenario: Generator bus voltages with preventive actions

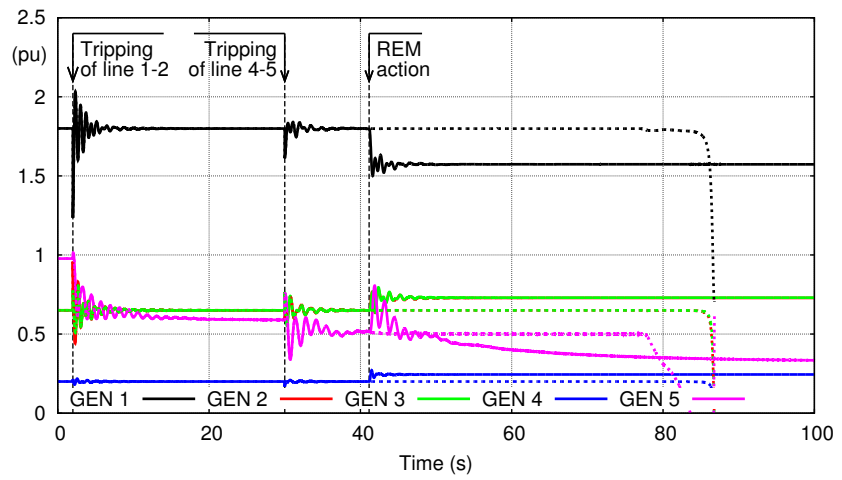

(b) Stabilized scenario: Generator power injection with preventive actions

Fig. 11. IEEE 14-bus: Results of the instable scenario, when applying the remedial action method

since it represents the connection to a strong external grid.

The method determined that the active power of G1 needs to be reduced by $22.4 \mathrm{MW}$ to bring the system back into a secure state. The computation of the power reserves of the remaining generators showed that only all three generators together can balance the needed power reduction. The contribution of each generator was determined from the size of its power reserve with respect to the available total power reserve. Table II shows the complete redispatch solution and that in this case the size of the machine rather than the maximum power injection was the limiting factor. Figure 11 shows the simulation results, when the proposed redispatch of active power is applied. Figure 11a shows the voltages at the generator buses over time and Fig. 11b shows the active power injection of the generators. In both graphs, the simulation results without corrective actions are illustrated with dashed lines and the results with the proposed redispatch with solid lines.

It can be seen that the collapse in voltage was prevented, due to the power redispatch, where the power output of G1 was reduced and the power injections of G2-G4 were increased. An additional benefit was the prevention of the third disturbance,

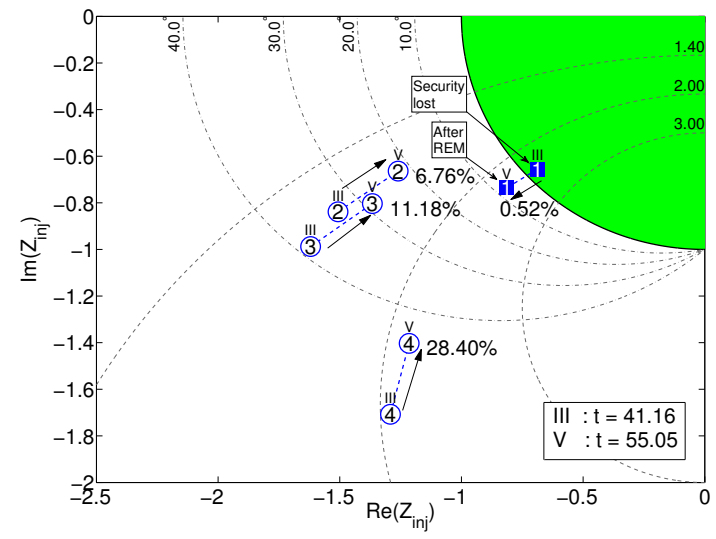

Fig. 12. IEEE 14-bus: Stability condition before and after preventive action, the percentages indicate the stability margin after the preventive action. The Arabic numbers refer to the number of the generator, e.g. "1" refers to G1.

which was the activation of the OEL of G1.

Figure 12 shows the stability condition of the system at the time when insecure operation was detected (see Fig. $10 \mathrm{III}$ and Fig. 12 III) and when a new quasi steady state was reached after the corrective redispatch ( Fig. $12 \mathrm{~V}$ ). The labels at the OPs show the stability margin of the corresponding generator.

The stability margins of the generators $\mathrm{G} 2-\mathrm{G} 4$ were reduced considerably and the stability margin of the $N-1$ OP of G1 was increased to a secure margin $(\geq 0.5 \%)$. Finally, the graph shows that all generators are secure and stable.

\section{B. Scenario 2: Actual OP crosses stability boundary}

1) Test System: The Nordic32 system [18] was chosen for the second test case. The system topology was modified as described in [5]. Moreover, in the presented case the initial condition from [18] were altered. The modifications are as follows: 1) Generator G22 is set out-of-service and its load is shared by G7, G8, G20 and G21. 2) Generator G7 is manually excited. 3) One line connecting 2031 and 2032 is disconnected. 4) Load alternations: at 41 increased by $20 \mathrm{MW}$, at 2031 increased by $10 \mathrm{MW}$ and at 2032 decreased by $10 \mathrm{MW}$.

In the presented case, the system is in a very stressed condition, where the excitation voltage of various generators is close to the respective limit.

2) Unstable scenario: In order to provoke instability and a collapse in voltage, the line connecting bus 4021 and 4042 was tripped at $\mathrm{t}=5 \mathrm{~s}$. The disturbance leads to electromechanical oscillations, which damp out, but lead to activation of various OELs before a collapse in voltage can be observed (see Fig. 13). The OELs are activated successively beginning with G11 at $18.28 \mathrm{~s}, \mathrm{G} 4$ at $23.70 \mathrm{~s}, \mathrm{G} 13$ at $33.11 \mathrm{~s}, \mathrm{G} 10$ at $42.15 \mathrm{~s}$, G12 at $45.90 \mathrm{~s}$ and $\mathrm{G} 6$ at $47.13 \mathrm{~s}$.

The ASSRAS assessment results are shown at a selection of time instances I-IV in Fig. 14. Here only the start and end OPs (I and IV) are depicted by big markers, which indicate the type of the OP, while the intermediate OPs at II and III are solely represented by small blue dots.

In the pre-fault condition seen in Fig. 14 I all the generators are stable and secure. It can be observed that the most critical generator is the manually excited generator G7 since its actual OP is relatively close to the stability boundary with a margin of $5.73 \%$. The remaining displayed OPs are $N-1$ OPs and, 


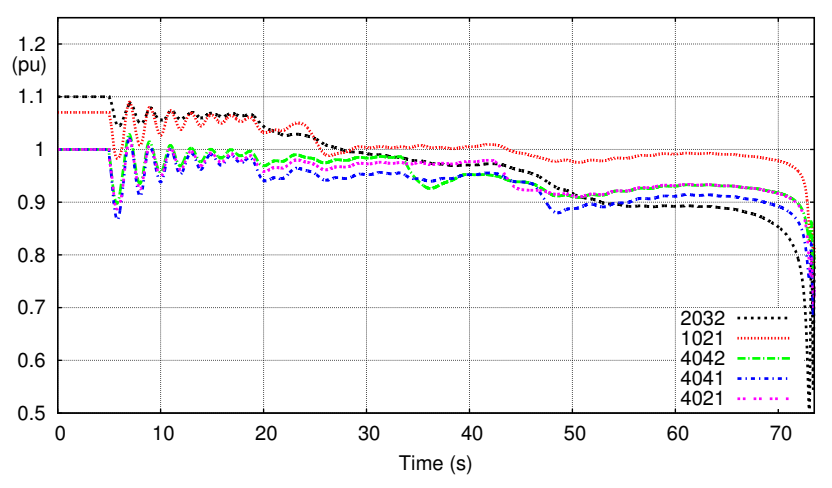

Fig. 13. Voltage magnitude at a selection of buses

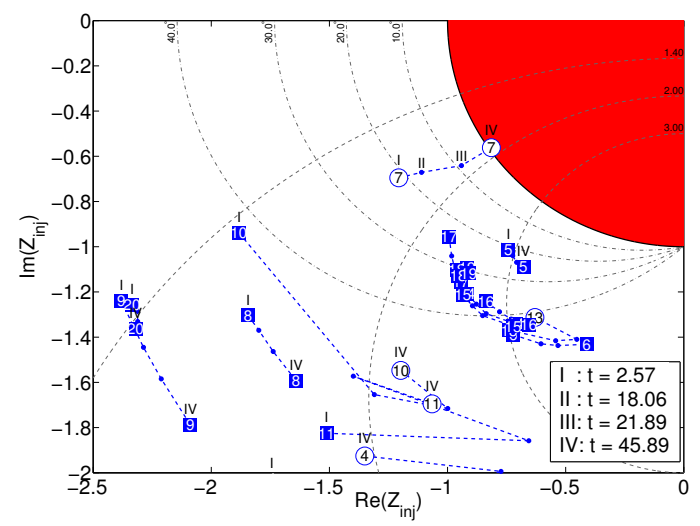

Fig. 14. Nordic32: Stability assessment results: Pre-fault $(2.5 \mathrm{~s})$, after first disturbance loss of line 4021-4042 (18.06 s), after second disturbance activation of OEL of G11 (21.89 s), G7 crosses stability boundary (45.89 s). Arabic numbers refer to the number of the generator, e.g. "1" refers to G1.

consequently, are less critical. However, these will become the actual OPs, when the OEL of the respective generator is activated. The loss of the transmission line connecting bus 4021 and 4042 reduced the stability margin of G7 to $3.68 \%$ (II) and its OP moved closer to the boundary. Afterwards, the OEL of G11 was activated at $18.28 \mathrm{~s}$ and, hence, the prior $N-1$ OP G11 now became the actual OP. This second disturbance led to a further reduction of the stability margin of the critical machine $\mathrm{G} 7$ to $0.98 \%$ (III). The subsequent activations of the OEL's of G4, G10 and G13 caused further depression of the stability margin of $\mathrm{G} 7$ and the generator eventually crossed the stability boundary at $44.98 \mathrm{~s}$, as shown in Fig. 14 IV. Afterwards, the generator started to drift away from the remaining generators, which eventually causes a collapse of the voltages at $\approx 73 \mathrm{~s} \mathrm{(see} \mathrm{Fig.} \mathrm{13).}$

The results show that the assessment method detects the imminent instability approximately $28 \mathrm{~s}$ before the collapse.

3) Scenario with remedial action: In this section, the same instability scenario as prior is investigated, but this time the remedial action method is executed, when a generator's stability margin falls below the trigger margin. Offline simulation of the test system showed, that the margin thresholds for actual OPs has to be chosen more conservative with $2 \%$ for the security margin and $1 \%$ for the trigger margin.

In the prior described scenario, the stability margin of generator $\mathrm{G} 7$ fell below the trigger margin at $21.89 \mathrm{~s}$ (see Fig. 14 III). The developed method determined the necessary remedial action to bring the system back into a secure state
TABLE III

ACTIVE POWER RE-DISPATCH SOLUTIONS

\begin{tabular}{|c|c|c|c|c|c|}
\hline $\begin{array}{c}\text { Time } \\
s\end{array}$ & $\begin{array}{c}\text { Critical } \\
\text { Gen. }\end{array}$ & $\begin{array}{c}\text { Margin } \\
\% \Delta P_{i n j}\end{array}$ & $\begin{array}{c}\text { Necessary } \\
\Delta P\end{array}$ & $\begin{array}{c}\text { Supp. } \\
\text { Gen. }\end{array}$ & $\begin{array}{c}\text { Margin } \\
\text { after red. }\end{array}$ \\
\hline \hline 21.89 & $\mathrm{G} 7$ & $0.98 \%$ & $8.25 \mathrm{MW}$ & $\mathrm{G} 11$ & $1.53 \%$ \\
\hline 27.05 & $\mathrm{G} 7$ & $0.81 \%$ & $9.44 \mathrm{MW}$ & $\mathrm{G} 11$ & $1.05 \%$ \\
\hline 49.46 & $\mathrm{G} 7$ & $0.83 \%$ & $9.09 \mathrm{MW}$ & $\mathrm{G} 11$ & $2.14 \%$ \\
\hline
\end{tabular}

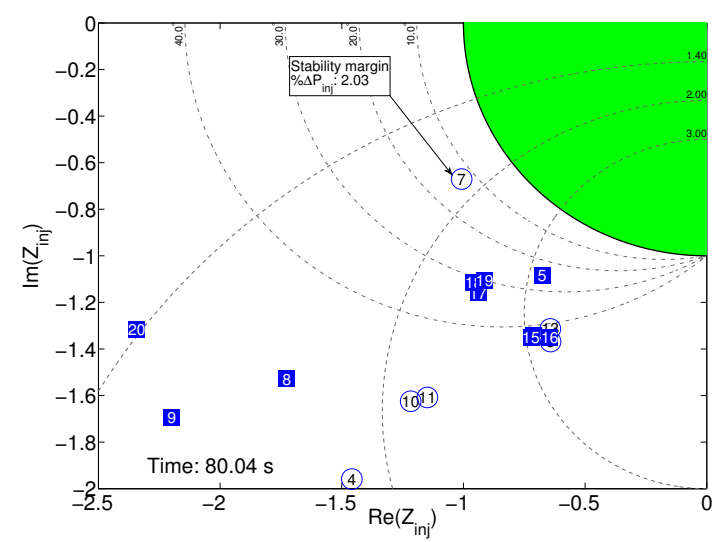

Fig. 15. Stability condition ca. $30 \mathrm{~s}$ after the last corrective redispatch. The Arabic numbers refer to the number of the generator, e.g. "1" refers to G1.

to be an active power reduction of G7 by $8.25 \mathrm{MW}$. Furthermore, the method computed the available power reserves of the remaining generators. G11 was chosen to counterbalance the power reduction with an equal increase, because of its electrical proximity and its sufficient power reserve. Due to the OEL activation of $\mathrm{G} 4$, the margin of $\mathrm{G} 7$ again fell below the trigger threshold, which in return executed the remedial action method and caused a second corrective power redispatch. The plurality of OEL activations caused G7 to cross the threshold a third time. Details on the three corrective actions can be found in Table III.

The table shows the time, when a corrective action is applied, the critical generator, whose power injection is reduced, as well as its stability margin and the needed power reduction. Furthermore, it shows the supporting generator, which will counterbalance the power reduction, and the stability margin of the critical generator after the corrective action. During the first two corrective actions, the system is in a state, where ULTC transformers and OELs are acting. Consequently, the assumption of quasi steady state conditions introduces an error and may explain, why the remedial action do not lead to a stability margin greater or equal to the chosen security margin. The last corrective redispatch succeeds to bring the system back into a secure state. Figure 15 depicts the system after the last redispatch and after a new quasi steady state was reached. It can be seen that all OPs are in the secure and stable region.

Figure 16 displays the voltage magnitudes at a selection of buses over time. The dashed lines are the voltages in the unstable case and the solid lines are the voltages in the case with corrective control actions. The graph shows that the corrective actions prevented the system collapse and led to a stabilization of the voltages.

\section{DisCussion}

In this paper a new patented method was presented that determines remedial actions to prevent a blackout. A real-time 


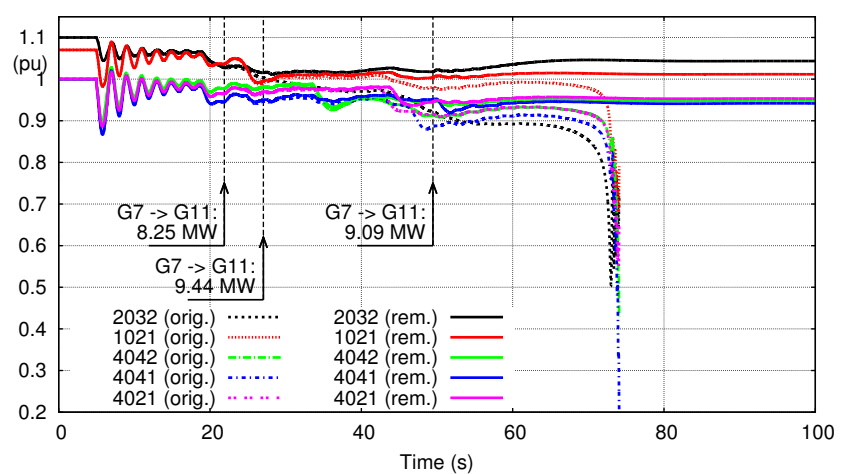

Fig. 16. Voltage magnitude at a selection of buses with corrective redispatch

ASSRAS assessment method is used to identify insecure or unstable system operation. The presented method utilizes these information to compute corrective power redispatch solutions. The method is based on algebraically derived expressions, which makes it very well suited for real-time application. The control actions move the $\mathrm{OP}$ of the critical generator back into the secure region, while ensuring that none of the supporting generators enters an insecure state.

The method's capability of avoiding a collapse in voltage and an imminent blackout was demonstrated with simulation results from two scenarios and two test systems (IEEE 14-bus and Nordic32). In both cases, the determined corrective redispatches stabilized the system and restored the stability margin of the critical machine to a secure level. This demonstrates accuracy and effectiveness of the proposed methodology.

In future work, the proposed approach could be further developed, e.g. taking into account additional constraints, such as the limits of the OELs, when determining the available active power reserves.

\section{REFERENCES}

[1] Danish energy agency, "Energy Policy in Denmark," Danish Energy Agency, Amaliegade 44, 1256 Copenhagen, Denmark, Tech. Rep. December, 2012.

[2] F. Li, W. Qiao, H. Sun, H. Wan, J. Wang, Y. Xia, Z. Xu, and P. Zhang, "Smart Transmission Grid: Vision and Framework," IEEE Transactions on Smart Grid, vol. 1, no. 2, pp. 168-177, Sep. 2010.

[3] M. Glavic and T. Van Cutsem, "Wide-Area Detection of Voltage Instability From Synchronized Phasor Measurements. Part I: Principle," IEEE Transactions on Power Systems, vol. 24, no. 3, pp. 1408-1416, Aug. 2009.

[4] M. Glavic and T. Van Cutsem, "Wide-Area Detection of Voltage Instability From Synchronized Phasor Measurements. Part II: Simulation Results," IEEE Transactions on Power Systems, vol. 24, no. 3, pp. 1417-1425, Aug. 2009.

[5] H. Jóhannsson, A. H. Nielsen, and J. Østergaard, "Wide-Area Assessment of Aperiodic Small Signal Rotor Angle Stability in Real-Time," IEEE Transactions on Power Systems, pp. 1-13, 2013.

[6] J. Wen, W.-H. E. Liu, P. L. Arons, and S. K. Pandey, "Evolution Pathway Towards Wide Area Monitoring and ProtectionA Real-World Implementation of Centralized RAS System," IEEE Transactions on Smart Grid, vol. 5, no. 3, pp. 1506-1513, May 2014.

[7] Z. Yao, V. R. Vinnakota, Q. Zhu, C. Nichols, G. Dwernychuk, and T. Inga-Rojas, "Forewarned Is Forearmed: An Automated System for Remedial Action Schemes," IEEE Power and Energy Magazine, vol. 12, no. 3, pp. 77-86, May 2014.

[8] Y. Makarov, S. Lu, X. Guo, J. Gronquist, P. Du, T. Nguyen, and J. Burns, "Wide Area Security Region," Pacific Northwest National Laboratory, Richland, Washington 99352, Tech. Rep. March, 2010.

[9] J. Ma, T. Wang, S. Wang, X. Gao, X. Zhu, Z. Wang, and J. Thorp, "Application of dual youla parameterization based adaptive wide-area damping control for power system oscillations," Power Systems, IEEE Transactions on, vol. 29, no. 4, pp. 1602-1610, July 2014.
[10] A. Heniche and I. Kamwa, "Assessment of two methods to select widearea signals for power system damping control," Power Systems, IEEE Transactions on, vol. 23, no. 2, pp. 572-581, May 2008.

[11] E. Dmitrova, A. Nielsen, H. Jóhannsson, and K. Johansen, Early Prevention Method for Power Systems Instability. Technical University of Denmark, Department of Electrical Engineering, 2013.

[12] T. Weckesser and H. Jóhannsson, "Method of determining remedial control actions for a power system in an insecure state," 2013, wO2013098184; G05D5/00; H02J3/24; H02J3/46

[13] H. Jóhannsson, J. Østergaard, and A. H. Nielsen, "Identification of critical transmission limits in injection impedance plane," International Journal of Electrical Power \& Energy Systems, vol. 43, no. 1, pp. 433-443, Dec. 2012.

[14] S. Sommer and H. Jóhannsson, Real-Time Thevenin Impedance Computation. IEEE, 2013, p. 6497824.

[15] H. Jóhannsson, "Development of early warning methods for electric power systems," Ph.D. dissertation, 2011.

[16] K. Visakha, D. Thukaram, and L. Jenkins, "Transmission charges of power contracts based on relative electrical distances in open access," Electric Power Systems Research, vol. 70, no. 2, pp. 153 - 161, 2004.

[17] University of Washington, "Power System Test Case Archive," Aug. 2012. [Online]. Available: http://www.ee.washington.edu/research/pstca/

[18] M. Stubbe, "Long-term dynamics-phase II (report of CIGRE Task Force 38.02.08)," CIGRE, Tech. Rep. March, 1995.

\section{BIOGRAPHIES}

Tilman Weckesser (M'12) received the M.Sc. degree in sustainable energy engineering from the Technical University of Denmark (DTU), Lyngby, in 2011. He is currently a Ph.D. student at the Centre for Electric Power and Energy (CEE), Department of Electrical Engineering, DTU.

His research interests are in the field of power system dynamics and stability with a focus on the development of fast stability assessment methods.

Hjörtur Jóhannsson (M'10) received the M.Sc. and the PhD degree in Electrical Engineering from DTU, in 2007 and 2011 respectively. He is currently a research consultant at CEE, DTU Electrical Engineering.

His research interests concern the development of methods that provide an early warning for instability in electric power systems, and power systems dynamics, stability and control.

Jacob Østergaard (M'95-SM'09) received the M.Sc. degree in electrical engineering from the DTU, Lyngby, in 1995. He was with the Research Institute of Danish Electric Utilities for 10 years. Since 2005 he has been Professor and Head of CEE at DTU Electrical Engineering.

His research interests cover smart grids with focus on system integration of renewable energy and distributed energy resources, control architecture for future power system, and flexible demand 